\title{
Towards an Aircraft with Reduced Lateral Static Stability Using Electric Differential Thrust
}

\author{
Eric Nguyen Van* \\ ISAE DCAS, Toulouse, France \\ ONERA DTIS, Toulouse France \\ Daniel Alazard ${ }^{\dagger}$ and Philippe Pastor ${ }^{\ddagger}$ \\ ISAE DCAS, Toulouse, France \\ Carsten Döll ${ }^{\S}$ \\ ONERA DTIS, Toulouse, France
}

\begin{abstract}
In the context of aircraft drag reduction, we study the possibility of reducing the area of the vertical tail using Distributed Electric Propulsion (DEP) while maintaining lateral stability with active Differential Thrust (DT). Distributed Electric Propulsion is usually thought of as a mean to increase aerodynamic efficiency by exploiting the beneficial effects of accelerating air around key parts of the aircraft. However, it can also be seen as a collection of actuation devices generating additional moments through Differential Thrust. When the engines are distributed along the lateral axis, the aircraft designer may take advantage of the increase of control authority on yaw to reduce the static stability or the control authority provided by the Vertical Tail (VT). This in turn would allow a reduction of vertical tail surface area. In order to explore and assess this idea, we suggest a framework to compare flight qualities of a traditional configuration versus a configuration using Distributed Electric Propulsion and Differential Thrust. The framework provides information on the flight envelop and stability of the aircraft by computing a map of the equilibrium. Thanks to a global approach, it allows to study any aircraft or DEP configurations in any flight phase. In addition, a key feature of the framework is the inclusion of the VeDSC[1] method to compute analytically the contribution of the vertical tail to lateral stability. It allows to study effects of a $30 \%$ reduction of VT surface area. Here are presented the first results and potential of using differential thrust to reduce the area of the vertical tail and the reasons for us to continue developing this framework.
\end{abstract}

\footnotetext{
*PhD, eric.nguyen-van@isae.fr, eric.nguyen_van@onera.fr

${ }^{\dagger}$ Professor Researcher, daniel.alazard@ @isae.fr.

†Professor Researcher, philippe.pastor@isae.fr.

${ }^{\S}$ Research engineer, carsten.doll@onera.fr.
} 


\section{Nomenclature}

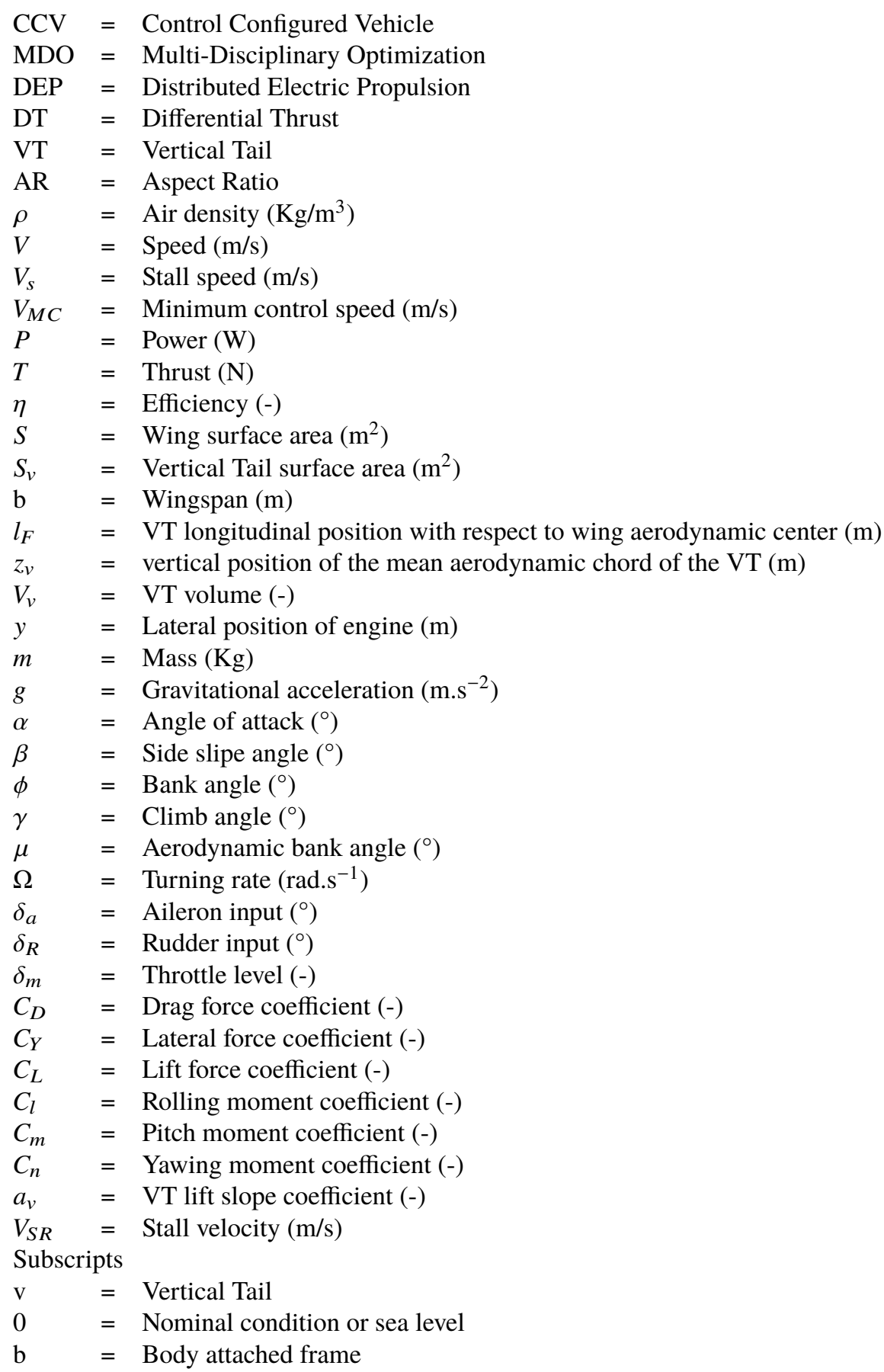

\section{Introduction}

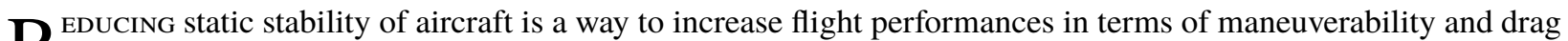
R reduction. The first case benefits fighter aircraft which are usually designed unstable by manipulating stability surfaces and position of the center of gravity. When reducing the tail volumes i.e stability surfaces and level arms, we also achieve a significant reduction in drag due to the diminution of wetted surfaces. In the civilian domain, this effect is increasingly exploited due to the important flight performance increase [2], [3].

To obtain a high level of performance improvement, the aircraft design strategy must be modified so as to include a 
stability and control block that can act on the geometry of the airplane. This way, the designer can take advantage of fly by wire and active control. This domain is called Control Configured Vehicle (CCV) and is not limited to airplane[3]. An example of aircraft design based on CCV principles has been describe by Anderson and Mason in [4]. The authors introduce the main problematic with CCV; the automation of control law design and flight quality assessment in order to embed the discipline in a Multi-Disciplinary Optimization MDO . They propose a solution based on fuzzy logic to include CCV in an MDO. In the following years B. Chudoba and H. Smith [5] as well as R. E. Perez and H. T. Liu [6], both presented an MDO framework with stability and control laws design based on stability augmentation system and pole placement technics, with B. Chudoba focusing on generic modeling and characterization. More recently a study from [7] shows a methodology for inclusion of stability and control using optimal control into MDO while Y. Denieul [8] shows in his thesis the integrated optimization of actuation surfaces together with the control law based on $H_{\infty}$ methods. All these examples tend towards a more global approach in aircraft design due to the coupling of two or more disciplines, here mainly flight performances and flight stability and control.

While methods exist for designing aircraft with relaxed longitudinal stability [9], the reduction of lateral static stability is bounded by stability requirements arising from emergency situations. The vertical stabilizer is dimensioned to handle single engine failure and/or strong lateral winds up to $25 \mathrm{kt}$ [10], [11], [12], [13], [14]. Usually the parameter constraining the size of the vertical stabilizer is the Minimum Control Speed : $V_{M C}$ defined by certification specifications as the minimum velocity at which, if an engine is made inoperative, the vertical tail has sufficient control authority to counter the yawing moment created by the remaining engine at maximum take off power within $5^{\circ}$ of bank angle [13]. This constraint is a hard limit for the reduction of the rudder, impacting the design for the whole Vertical Tail (VT). In addition as Morris suggests in [14], the VT is often over-dimensioned to cope with non-linear viscous effects such as masking by the fuselage. A lack of adapted tool able to predict the performance of the VT in these conditions is called responsible for this case.

Efforts for reducing the fin are taken both in research institutions and aircraft industry. They include better performance prediction for design phase [15] as well as flow control to increase fin efficiency [16]. In this last example, a possible reduction of the fin area could amount to $15 \%$ with a total drag reduction of $0.9 \%$.

We envision to increase this reduction by using a new game changing technology that has recently gained interest among aircraft propulsion system; Distributed Electric Propulsion and differential thrust. Differential thrust is already used in current multi-engine aircraft in case of complete loss of hydraulic system as a mean to control flight. In this case, it is called Propulsion Controlled Aircraft [17], a system developed first at NASA Dryden center. However, it cannot be used as a mean to increase lateral stability because of the lack of engine redundancy and of the large reaction time of turbo-machines.

Recently, more and more interest has been held on electric airplanes. Though studies usually focus on the challenge of energy storing, we will focus and exploit the characteristics of electric engines. These ones are our actuators for differential thrust. The reader is referred to [18] for a complete list of electric engine advantages. We will retain only the following; scale free efficiency and high power density. They allow to rethink the way of airframe propulsion integration. The designer can freely place the engines where they may increase aerodynamic performances. Two effects are usually seeked: 1.Increase maximum lift by blowing and 2.Boundary Layer Ingestion for drag reduction [19], [20]. This is the case for the all electric concept plane AMPERE from ONERA [21] where efans are integrated to the wing to benefit from blowing effects and NASA aircraft X57[22]. For this last example the distributed engines are expected to replace high lift devices. The advantages for lateral control with differential thrust are threefold:

- Redundancy : if one or more critical engines are suddenly made inoperative, only a small portion of the thrust is lost. There remain an important number of engines to reallocate the thrust.

- Reaction time: with small electric engines the reaction time can be of the order of $10^{-1} \mathrm{~s}$ [23], therefore fast with respect to aircraft flight dynamics.

- Airframe integration: the engines being distributed along the wing (in the previously mentioned airplanes), we benefit from an important level arm without adding specific structures.

Though the idea has been suggested in [20], example and study of differential thrust for yaw control and relaxed lateral stability has, to the best of the authors knowledge, not yet received much attention. Hence, the goal of this study is to explore the possibilities of relaxing lateral stability by using differential thrust with electric propulsion and more specifically the requirements in terms of design.

A detailed treatment of the distributed propulsion is not the approach retained for this study. Because electric propulsion is easily integrated into airframe, important synergies can exit between aerodynamics, structure, flight control and propulsion. This multi-physic environment necessitate a global approach to study DEP aircraft.

Instead, a framework is proposed to assess different configurations of DEP aircraft starting with a simple model of 
distributed propulsion. Only point force produced by engines equipped with propellers evenly positioned on the wing are considered for this study. Any interaction between slipstreams and airframe will be taken into account at a later stage once the framework is ready for increasing complexity.

A way of comparing the flight qualities of two different aircraft has been proposed by Goman in [24] by computing and comparing the flight envelop of the two aircraft. This method relies on finding equilibrium and allows to assess the stability of an aircraft. It is also possible to take into account a stability augmentation in the computation for an unstable aircraft. We selected this method and augmented it to take into account differential propulsion as well.

The present paper is organized as follow, first the mathematical treatment of the equilibrium search is performed with the distributed propulsion model. Then, it is explained how the aerodynamic database is constructed to study the variation of vertical tail area. Finally results obtained with this tool and the potential of differential thrust over traditional configuration are shown.

\section{Static equilibrium}

In this section we explain the mathematical fundamentals as well as the model used to capture differential thrust. The equation of flights in aerodynamic frame are first re-called, then modeling of propulsion is shown and finally the trimming algorithm is explained.

\section{A. Equation of flight}

The equations of flight in the aerodynamic frame, assuming uniform wind velocity are used. This form is the most commonly used when studying the lateral flight dynamics. Equations are taken from Boiffier [25] and are equivalent to those presented by Goman in[24]:

$$
\begin{aligned}
\left(\begin{array}{c}
m \dot{V} \\
m[\dot{\beta} V-V(p \sin \alpha-r \cos \alpha)] \\
m[\dot{\alpha} V \cos \beta+V(\sin \beta(p \cos \alpha+r \sin \alpha)-q \cos \beta)]
\end{array}\right) & =m g\left(\begin{array}{c}
-\sin \gamma \\
\cos \gamma \sin \mu \\
\cos \gamma \cos \mu
\end{array}\right)+\frac{1}{2} \rho S V^{2}\left(\begin{array}{c}
-C_{D} \\
C_{Y} \\
-C_{L}
\end{array}\right)+\mathbf{H}_{\mathbf{a b}}\left(\begin{array}{l}
F_{x_{b}} \\
F_{y_{b}} \\
F_{z_{b}}
\end{array}\right) \\
\mathbf{I}\left(\begin{array}{c}
\dot{p} \\
\dot{q} \\
\dot{r}
\end{array}\right)+\left(\begin{array}{l}
p \\
q \\
r
\end{array}\right) \times \mathbf{I}\left(\begin{array}{l}
p \\
q \\
r
\end{array}\right) & =\frac{1}{2} \rho S V^{2} l\left(\begin{array}{c}
C_{l} \\
C_{m} \\
C_{n}
\end{array}\right)+\left(\begin{array}{c}
M_{x_{b}} \\
M_{y_{b}} \\
M_{z_{b}}
\end{array}\right)
\end{aligned}
$$

Where $\mathbf{I}$ is the inertia matrix, $\mathbf{H}_{\mathbf{a b}}$ is the rotation matrix from body to aerodynamic frame projecting forces $\left(F_{x_{b}}, F_{y_{b}}, F_{z_{b}}\right)$ due to propulsion:

$$
H_{a b}=\left(\begin{array}{ccc}
\cos \alpha \cos \beta & \sin \beta & \sin \alpha \cos \beta \\
-\cos \alpha \sin \beta & \cos \beta & \sin \alpha \sin \beta \\
-\sin \alpha & 0 & \cos \alpha
\end{array}\right)
$$

The weight is projected onto the aerodynamic airframe through the climb angle $\gamma$ and the aerodynamic bank angle $\mu$. These terms can be expressed as [25]:

$$
\begin{aligned}
& \cos \gamma \sin \mu=\sin \theta \cos \alpha \sin \beta+\cos \beta \cos \theta \sin \phi-\sin \alpha \sin \beta \cos \theta \cos \phi \\
& \cos \gamma \cos \mu=\sin \theta \sin \alpha+\cos \beta \cos \theta \cos \phi
\end{aligned}
$$

The complementary kinematic equations are :

$$
\left(\begin{array}{c}
\dot{\phi} \\
\dot{\theta} \\
\dot{\psi}
\end{array}\right)=\left(\begin{array}{ccc}
1 & \sin \phi \tan \theta & \cos \phi \tan \theta \\
0 & \cos \phi & -\sin \phi \\
0 & \frac{\sin \phi}{\cos \theta} & \frac{\cos \phi}{\cos \theta}
\end{array}\right)\left(\begin{array}{l}
p \\
q \\
r
\end{array}\right)
$$

Two additional parameters are defined to find pseudo-equilibrium: the climb angle $\gamma$ and the turn rate $\Omega$, which for steady turns is simply equal to the rate of change of heading:

$$
\begin{aligned}
\sin \gamma & =\cos \alpha \cos \beta \sin \theta-\sin \beta \sin \phi \cos \theta-\sin \alpha \cos \beta \cos \phi \cos \theta \\
\Omega & =\dot{\psi}
\end{aligned}
$$


For steady turn rates, $\dot{\psi} \neq 0$ and one can find a pseudo-equilibrium by imposing the turn rate $\Omega$ as a parameter :

$$
\Omega=(q \sin \phi+r \cos \phi) \frac{1}{\cos \theta}
$$

Leaving the heading angle aside, equations (17), (2), (6), (7) and (9) represent a set of $N_{e}=10$ equations to satisfy. The state vector is $\mathbf{x}=[V, \alpha, \beta, p, q, r, \phi, \theta]$ and counts $n_{x}=8$ variables. The input vector corresponding to control surfaces, respectively ailerons, elevator and rudder is $\mathbf{u}=\left[\delta_{a}, \delta_{e}, \delta_{R}\right]$ with $n_{u}=3$ variables. Finally we count $n_{p}=2$ additional parameters $\gamma$ and $\Omega$. The model is now ready to be complemented with a model of distributed propulsion.

\section{B. Propulsion modeling}

Propulsion and differential thrust are modeled together and are assumed to only contribute to generating forces and moments. Meaning that the rotor terms or gyroscopic effects are let aside such that equations of flight aren't further modified. By summing the thrust forces and moments based on the geometrical arrangement shown in Fig 1 :

$$
\left(\begin{array}{c}
F x_{b} \\
F y_{b} \\
F z_{b}
\end{array}\right)=\left(\begin{array}{c}
\sum_{i=1}^{N} T_{x, i} \\
0 \\
0
\end{array}\right) \text { and } \quad\left(\begin{array}{l}
M x_{b} \\
M y_{b} \\
M z_{b}
\end{array}\right)=\left(\begin{array}{c}
0 \\
0 \\
\sum_{i=1}^{N}-T_{x, i} y_{i}
\end{array}\right)
$$

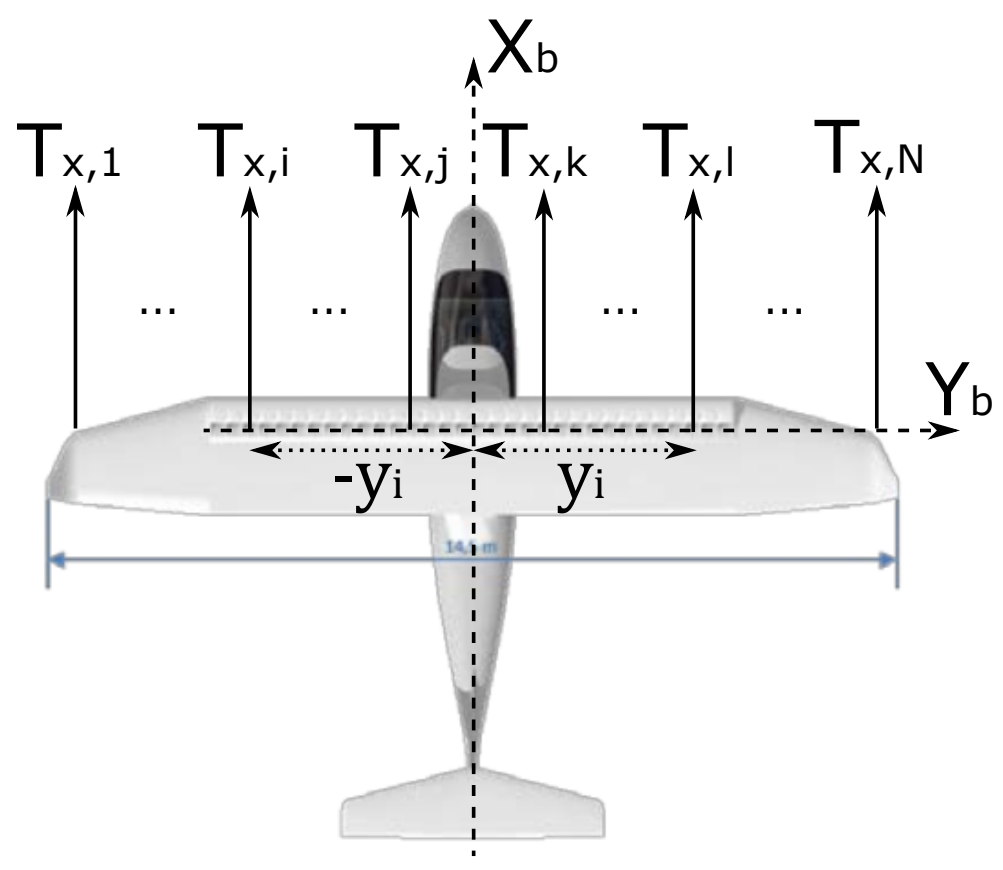

Fig. 1 Illustration of thrust distribution.

Point forces are considered symmetrically placed along the wing. Unlike the configuration of Ampere [21], equal repartition of engine from root to wing-tip is assumed.

Where $T_{x, i}$ and $y_{i}$ are respectively the thrust force and distance between the body $\mathrm{X}$ axis and the $\mathrm{i}^{\text {th }}$ motor. Our study has previously been limited to propulsive system based on electric engine running propellers. For aircraft equipped with a propeller or a fan, a common thrust model can be [26]:

$$
T=P V^{-1} \eta_{p} \frac{\rho}{\rho_{0}} \delta x
$$

Where $\mathrm{P}$ is the engine power at sea level, $\mathrm{V}$ is the flight velocity, $\eta_{p}$ is the propeller or fan efficiency, $\rho$ the air density and $\delta_{x}$ the throttle command. Equation (11) models the loss of power of air breathing engines with variation of air density. 
Electric motors on the contrary, do not suffer from rarefaction of air as turbomachines, such that we may conciser the following thrust model for computing $T_{x, i}$ [26]:

$$
T_{x, i}=\frac{P_{E}}{N} V^{-1} \eta_{m} \eta_{p} \delta_{x, i}
$$

With $P_{E}$ being the total electrical power available from the line, $N$ being the total number of engine, $\eta_{m}$ and $\eta_{p}$ respectively the engine and propeller efficiency (both considered constant). Hence, we concider that the power is equally divided between each engine. Finally, $\delta_{x, i}$ is the throttle command of each engine and is added to the control input vector : $\mathbf{u}=\left[\delta_{a}, \delta_{e}, \delta_{R}, \delta_{x, 1}, \ldots, \delta_{x, N}\right]$. The number of input becomes : $n_{u}=N_{m}+3$.

One may argue that electric propulsion will be impacted by altitude anyway. It is true in many aspects, for example:

- With increasing altitude cooling of electric engine and power electronics can become more difficult

- Maximum rated voltage of conductors decreases with altitude due to Corona effect [27]

- Finally, in the case of series hybrid or turbo-electric propulsion, the turbo-machine producing the electric power remains sensible to air rarefaction.

These limitations are either due to technological locks that can be overcome with increasing interest in electric propulsion or associated with a level of details outside the scope of our study. For these reasons, we will keep the assumption that engine power is constant with altitude.

\section{Finding the trim position by optimization}

Similarly to what is done in [24], a set of additional constraints $N_{c}$ can now be defined to condition the problem such that only one possible solution exists. The number of variables to determine $\left(n_{x}+n_{u}+n_{p}\right)$ must equal the number of equations and constraints $N_{e}+N_{c}$. In this case, considering that the number of engine varies, the number of additional constraints to define is given by:

$$
\begin{aligned}
& N_{c}=n_{x}+n_{u}+n_{p}-N_{e} \\
& N_{c}=N_{m}+3
\end{aligned}
$$

In the case where differential thrust is not used, the additional input is $N_{m}=1$, representing forward thrust or throttle level, and one must fix $N_{c}=4$ additional constraints, similarly as in [24]. These additional constraints are added to determine the flight condition, typically fixing the following variables: $[V, \beta, \gamma, \Omega]$. With differential propulsion, the minimum number of additional input is $N_{m}=2$ with two engines. Consequently, the problem becomes quickly over-determined. An infinite number of equilibrium points can exist. This can be pictured by the different possible combination of throttle level and rudder action to satisfy a certain thrust and yaw moment.

For over-determined problems, it is common to use optimization methods to find a satisfying solution [28]. Two options are possible: one may define the input vector as $\mathbf{u}=\left[\delta_{a}, \delta_{e}, \delta_{n}, \delta_{x}\right]$ with $\delta_{n}$ being the total yaw moment input and $\delta_{x}$ the total thrust force such that the equilibrium problem is well conditioned and then use an optimization method to find the $\delta_{R}$ and $\delta_{x, i}$. Or one could run the optimization on the complete set of variables. We selected the second option for this study. This is motivated by the will of maintaining a tight coupling between flight dynamics and differential thrust.

It should be stressed that the first option leaves room for development of

- Generic control and allocation laws

- Optimal control

- Optimisation of design

These aspects will be the object of future studies.

Without loss of generality, additional higher and lower bounds are added on control inputs, angle of attack and bank angle depending on the flight phase. For example, the bank angle is limited to $\pm 5^{\circ}$ when studying engine failure at take off as stated by flight regulation [13]. These bounds are resumed in table 1 and are in part, dependent on the aircraft selected for the study.

The following variables are chosen to imposed the flight conditions $[V, \beta, \gamma, \Omega]$. The objective function to minimize is defined as the power required to maintain equilibrium. Such an objective function makes sense in the point of view of the designer who will look for minimizing the power to install on the aircraft. Finally, to simulate engine failure we simple add a constraint on the throttle level of the corresponding engines. 
The problem hence writes:

$$
\begin{aligned}
& \min _{\tilde{x}} \sum_{i=1}^{N} T_{x, i} V \\
& \text { With: } \quad \tilde{x}=\left[\alpha, p, q, r, \phi, \theta, \delta_{a}, \delta_{e}, \delta_{R}, \delta_{x, 1}, \ldots, \delta_{x, N}\right] \\
& \text { Subjected to: } \\
& \left(\begin{array}{c}
0 \\
-m V(p \sin \alpha-r \cos \alpha) \\
m V[\sin \beta(p \cos \alpha+r \sin \alpha)-q \cos \beta]
\end{array}\right)=m g\left(\begin{array}{c}
-\sin \gamma \\
\cos \gamma \sin \mu \\
\cos \gamma \cos \mu
\end{array}\right)+\frac{1}{2} \rho S V^{2}\left(\begin{array}{c}
-C_{d} \\
C_{Y} \\
-C_{L}
\end{array}\right)+\mathbf{H}_{\mathbf{a b}}\left(\begin{array}{c}
\sum_{i=1}^{N} T_{x, i} \\
0 \\
0
\end{array}\right) \\
& 0=\frac{1}{2} \rho S V^{2} l\left(\begin{array}{c}
C_{l} \\
C_{m} \\
C_{n}
\end{array}\right)+\left(\begin{array}{c}
0 \\
0 \\
\sum_{i=1}^{N} T_{x, i} y_{i}
\end{array}\right)-\left(\begin{array}{c}
p \\
q \\
r
\end{array}\right) \times \mathbf{I}\left(\begin{array}{c}
p \\
q \\
r
\end{array}\right) \\
& 0=p+q \sin \phi \tan \theta+r \cos \phi \tan \theta \\
& 0=q \cos \phi-r \sin \phi \\
& \Omega=(q \sin \phi+r \cos \phi) \sec \theta \\
& \sin \gamma=\cos \alpha \cos \beta \sin \theta-\sin \beta \sin \phi \cos \theta-\sin \alpha \cos \beta \cos \phi \cos \theta \\
& 0=\delta_{x, 1} \\
& \vdots \\
& 0=\delta_{x, j}
\end{aligned}
$$

The problem being now defined, the next section will detail the construction of the aerodynamic database as a function of VT area and flight phase.

Table 1 Additional bounds depending on flight phase

\begin{tabular}{l|c|c|c} 
Flight phase & $V<71 m \cdot s^{-1}$ & $V>71 m . s^{-1}$ & Engine failure \\
\hline$\alpha\left(^{\circ}\right)$ & $-2 \leq \alpha \leq 10$ & $-2 \leq \alpha \leq 15$ & $(-)$ \\
$\phi\left(^{\circ}\right)$ & \pm 30 & \pm 30 & \pm 5 \\
$\theta\left(^{\circ}\right)$ & \pm 30 & \pm 30 & \pm 30 \\
$\delta_{a}\left(^{\circ}\right)$ & \pm 20 & \pm 20 & \pm 20 \\
$\delta_{e}\left(^{\circ}\right)$ & \pm 20 & \pm 20 & \pm 20 \\
$\delta_{R}\left({ }^{\circ}\right)$ & \pm 25 & \pm 25 & \pm 25 \\
$\delta_{x, i}$ & $0<\delta_{x, i} \leq 1$ & $0<\delta_{x, i} \leq 1$ & $0<\delta_{x, i} \leq 1$
\end{tabular}




\section{Aerodynamic database}

In this section, it is explained how the aerodynamic database was obtained to study the effect of varying the vertical tail surface area. Assumptions are re-called to put the focus only on relaxed lateral stability.

\section{A. Reference Aircraft}

The method developed up to now is generic and could be used with any aircraft given the corresponding aerodynamic characteristics. To study the differences between a traditional configuration and a DEP aircraft, a baseline aircraft representing the class of aircraft most probable for electric propulsion and distributed propulsion has to be selected. Commuter aircraft are often cited as the next big step in developing electric airplanes since most of their mission are within the limits of electric propulsion in terms of endurance [18] [29]. These aircraft usually are equipped with turboprop engine and fly at subsonic velocity. A good representative of this class of aircraft is the ATR72 which details are reported in table 2

Table 2 ATR 72 general details [30], [31]

\begin{tabular}{l|c} 
Variables & Value \\
\hline Wingspan & $27 \mathrm{~m}$ \\
Wing surface area & $61 \mathrm{~m}^{2}$ \\
VT surface area & $12 \mathrm{~m}^{2}$ \\
Engine level arm & $4.1 \mathrm{~m}$ \\
Masse & $21500 \mathrm{Kg}$ \\
Total available power & $4000 \mathrm{KW}$ \\
Stall velocity $V_{s}$ & $50.5 \mathrm{~m} / \mathrm{s}$
\end{tabular}

So far, special care was given to keep the analysis as generic as possible to be able to compare flight qualities of different aircraft configurations. Following the same philosophy it is assumed no change in the geometry, mass or power available of the reference ATR72 between the baseline configuration and the electric propelled one.

The only feature allowed to change is the vertical tail which will be reduced so as to obtain relaxed lateral stability or a lightly unstable aircraft. Consequently, we will analyze a weakly unconventional aircraft configuration.

\section{B. Building the Aerodynamic Database}

For unconventional configurations, it is necessary to carefully select the method with which one can establish the aerodynamic database. As Chudoba explains in [32], the means of calculating aerodynamic characteristics can be organized in three categories summurised in Table 3

Table 3 Methods for Aerodynamic Analysis [5]

\begin{tabular}{l|c} 
Category & Example of methods \\
\hline Analytical & Lifting Line, Swept Wing Theory, ... \\
Empirical, semi-empirical & DATCOM, ESDU, ... \\
Numerical & Vortex Lattice Method, Panel Method, CFD, ...
\end{tabular}

Both analytical and empirical/semi-empirical methods are built on experiences and analysis of conventional configurations. Therefore there can hardly be considered as generic methods. Numerical methods offer different level of fidelity allowing to capture the specificity of unconventional design. For preliminary designs, VLM or Panel Method, both linear technics, are often preferred over CFD for their favorable precision relative to computational cost.

Although our configuration is only weakly unconventional, capturing the effect of geometrical changes in VT isn't something that analytical of empirical/semi-empirical methods can do because of the important influence of other aircraft components on the flow impacting the VT. This has been demonstrated by Nicolosi in [12], where his research team investigated the differences obtained between DATCOM, ESDU estimation technics and CFD methods complemented with wind tunnel experiments. 
The main contributors to flow perturbation impacting the VT are the fuselage and the horizontal tail which are acting as end plates, reducing the downwash at the root and tip of the vertical tail.

Using numerous CFD simulations complemented with wind tunnel test on a generic commuter aircraft model, Nicolosi and his research team could establish a new semi-empirical method called VeDSC. This method focuses on predicting the VT efficiency as a function of VT geometry and aircraft components. The main assumption is the fact that contribution of each components of the aircraft on lateral coefficients: $\left\{C_{Y}, C_{l}, C_{n}\right\} \equiv C_{\text {lat }}$, can be decoupled in the following way:

$$
C_{\mathrm{lat}_{\beta}}=C_{\mathrm{lat}, F_{\beta}}+C_{\mathrm{lat}, W_{\beta}}+C_{\mathrm{lat}, v_{\beta}}
$$

Where subscripts $F, W, v$ refer respectively to fuselage, wing and vertical tail and $C_{\cdot \beta}=\frac{\partial C}{\partial \beta}$. It is also assumed that the contribution of the VT, $C_{\text {lat, } v_{\beta}}$ is influenced by the fuselage, wing and horizontal tail but does not influence the other coefficients $C_{l a t, F_{\beta}}$ and $C_{l a t, W_{\beta}}$. The VeDSC method furnishes a way to estimate the coefficient $C_{\mathrm{lat}, v_{\beta}}$ through a reformulation of the VT lift slope coefficient defined as follow:

$$
a_{v}=K_{F} K_{W} K_{H} C_{L, v_{\beta}}
$$

With $C_{L, v_{\beta}}$ the lift slope of a swept wing determined using Diederich formula for swept wing [33]. $K_{F}, K_{W}$ and $K_{H}$ are corrective coefficients taking into account respectively the fuselage, wing and horizontal tail effects. The reader is referred to [34] and [35] for the complete formulation of these parameters.

The VT contribution to lateral coefficients is then calculated using formulas given by Etkin [36]:

$$
\begin{array}{llr}
C_{Y_{\beta}}=a_{v} \frac{S_{v}}{S}\left(1-\frac{\partial \sigma}{\partial \beta}\right) & C_{l_{\beta}}=-a_{v} \frac{S_{v} z_{v}}{S b}\left(1-\frac{\partial \sigma}{\partial \beta}\right) & C_{n_{\beta}}=a_{v} V_{v}\left(1-\frac{\partial \sigma}{\partial \beta}\right) \\
C_{Y_{p}}=-a_{v} \frac{S_{v}}{S}\left(2 \frac{z_{v}}{b}-\frac{\partial \sigma}{\partial \hat{p}}\right) & C_{n_{p}}=a_{v} V_{v}\left(2 \frac{z_{v}}{b}-\frac{\partial \sigma}{\partial \hat{p}}\right) \\
C_{Y_{r}}=a_{v} \frac{S_{v}}{S}\left(2 \frac{l_{F}}{b}+\frac{\partial \sigma}{\partial \hat{r}}\right) & C_{l_{r}}=a_{v} \frac{S_{v} z_{v}}{S b}\left(2 \frac{l_{F}}{b}+\frac{\partial \sigma}{\partial \hat{r}}\right) & C_{n_{r}}=-a_{v} V_{v}\left(2 \frac{l_{v}}{b}-\frac{\partial \sigma}{\partial \hat{r}}\right)
\end{array}
$$

This semi-empirical methods required hundreds of CFD simulations to explore a wide variety of parameter changes. Variation and validity intervals of some parameters of interest are shown in Table 4. The method could be extrapolated to VT of aspect ratio from 0.5 to 4 since the Diederich formula is valid for these values however the correcting terms would be out of interpolation range.

Overall the VeDSC method turns to be an adequate semi-empirical method to be used with our reference aircraft. In addition, having a formula to estimate the VT efficiency instead of running a VLM is an important time saving aspect.

In order to establish our aerodynamic database, we modeled the ATR72 without its VT into VSPaero using publicly available data essentially from [31]. The VLM included in VSPaero is then used to establish the longitudinal coefficients and contribution to the lateral coefficients. The contribution of the fuselage and wing to lateral coefficients has been found to be adequately estimated by the VLM. It is then complemented with the VeDSC method to quickly and accurately account for the changes in VT geometry. This renders the database extremely agile since we only need to run VLM simulations once.

Two ways of modifying the vertical tail are available. The first is the change of surface area while maintaining the aspect ratio constant. This gives linearly varying coefficients as it can be seen in Fig 2. The second comes from the fact that one may want to keep the horizontal tail high to avoid the slipstream of the propellers, hence the surface should be reduced without modifying the span. In turn the aspect ratio is increased up to reasonable values to limit extrapolation as shown in Fig 3 . This variation induces non linear variation of the coefficient and may be find useful for flight qualities.

Finally, to explore the flight performances in the whole flight envelop, it has been chosen to use a database in function of mach number as only flight parameter. For low speed flight, when the flaps are used, the parameters are assumed to be constant. Then, they vary with the mach number. The evolution of some coefficients with Mach number is illustrated in Fig 4. The Mach number 0.2 taken at see level represents the minimum flight velocity of the ATR72 without flaps. 


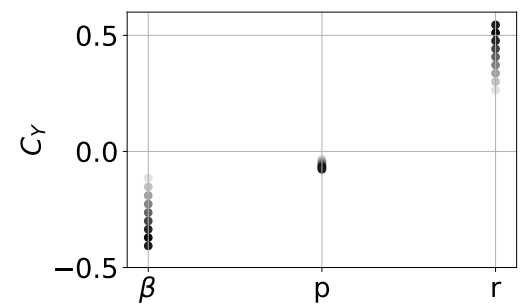

(a) All derivatives per rad

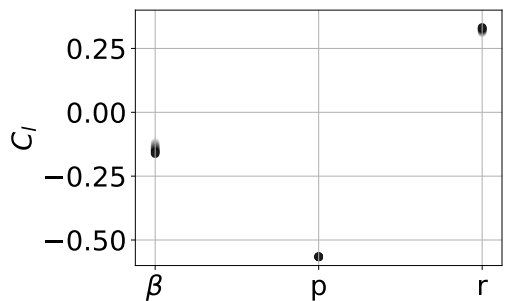

(b) All derivatives per rad

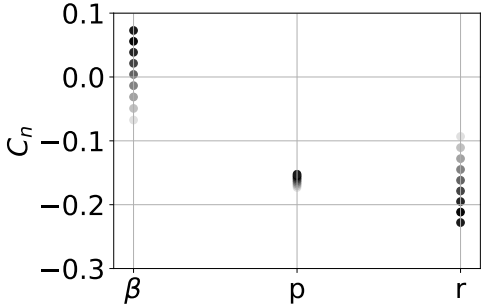

(c) All derivatives per rad

Fig. 2 Evolution of lateral coefficients of total aircraft with variation of VT area for constant AR. Whitest marker represents $S_{v}=0.1 S_{v, 0}$, darkest represents $S_{v}=S_{v, 0}$, per step of $0.1 S_{v, 0}$.

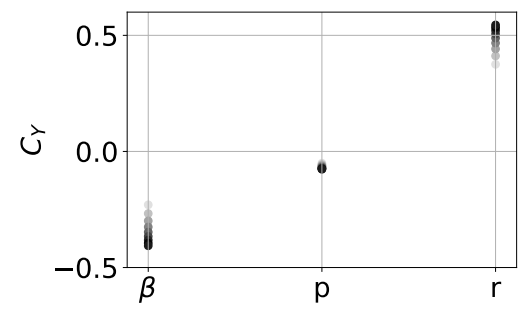

(a) All derivatives per rad

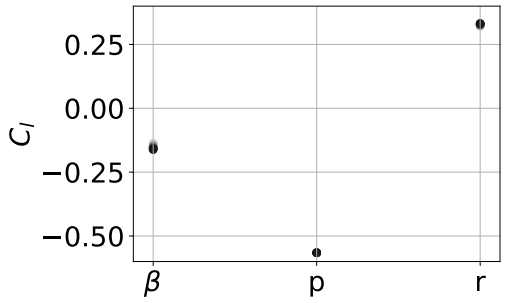

(b) All derivatives per rad

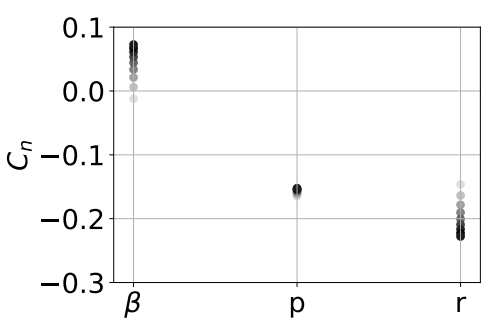

(c) All derivatives per rad

Fig. 3 Evolution of lateral coefficients of total aircraft with variation of VT area for constant span.

Whitest marker represents $S_{v}=0.3 S_{v, 0}$, darkest represents $S_{v}=S_{v, 0}$, per step of $0.1 S_{v, 0}$ The interval of AR swept is $[1.56,5.21]$

Table 4 A few parameter ranges on which VeDSC has been constructed. From [1]

\begin{tabular}{l|l|c} 
Parameters & Description & Range \\
\hline$A_{v}$ & VT aspect ratio & {$[1,2]$} \\
$A_{W}$ & Wing aspect ratio & {$[6,16]$} \\
$z_{W}$ & Wing vertical position, relative to fuselage centerline & {$[-1,1]$} \\
$z_{H}$ & Horizontal Tail Vertical Position, relative to VT span & {$[0,1]$} \\
$\frac{S_{H}}{S_{v}}$ & Relative Horizontal tail surface area & {$[0.5,2]$}
\end{tabular}




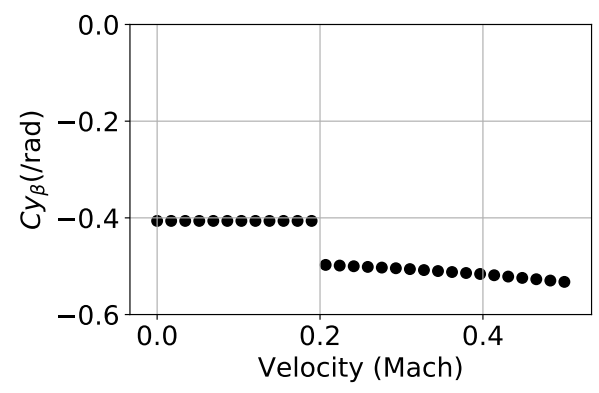

(a)

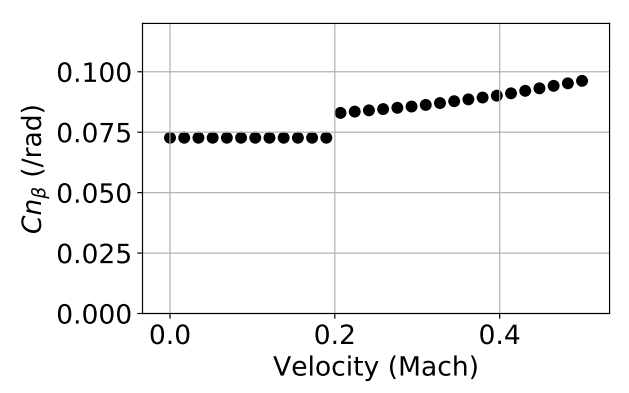

(c)

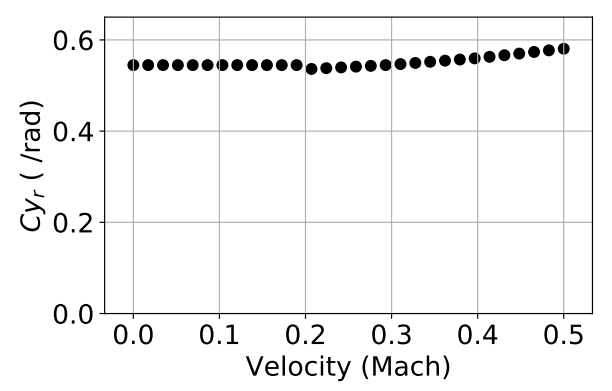

(b)

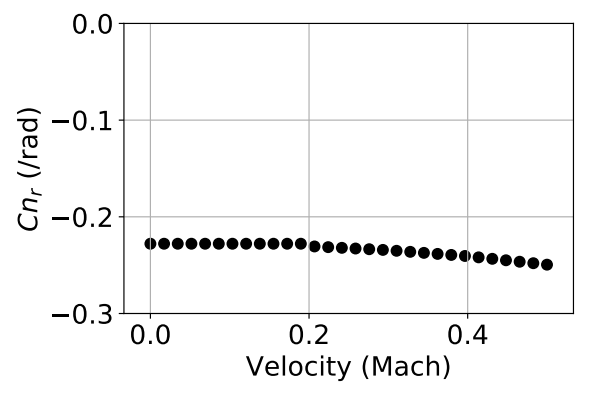

(d)

Fig. 4 Evolution of lateral coefficients of total aircraft with Mach number. 


\section{Results}

The framework detailed before is used to generate the results presented in this section. First, the case study is explained as well as the format used to present the results. Then an interpretation of the results is proposed.

\section{A. Case study}

At this point, it is possible to study a wide variety of flight configuration and situations. To keep the study concise we will limit our analysis to the situation of engine failures at take off with landing gear retracted as described by CS25.121, CS25.147 and CS12.149 [13]. These certification rules set the requirements on climb angle, directional control and $V_{M C}$ in case of one or multiple engine failures. Although this manner bounds the study to only one flight phase, a good insight is given on the tool efficiency to compare aircraft configuration and potential of differential thrust.

The certification rules indicate different flight conditions for twin engine and aircraft with more than four engines. The main differences are resumed in table 5

Table 5 Certification requirements for twin-engines and more than four engines [13]

\begin{tabular}{l|l|c} 
Parameters & Twin engine & More than four engines \\
\hline Gradient of climb, critical engine inoperative & $\leq 2.4 \%$ & $\leq 3.0 \%$ \\
Heading change of $15^{\circ}$ at $1.3 V_{S R_{1}}$ & One engine inoperative & Two critical engines inoperative \\
$V_{M C}<1.13 V_{S R}$ & One engine inoperative & One critical engine inoperative
\end{tabular}

It is of interest to investigate the most unfavorable situation for differential thrust which is clearly at a $3 \%$ climb angle and two or more critical engines inoperative.

For aircraft with more than four engines, the certification specifies that sufficient control must remain possible with the two critical engines inoperative at $1.3 V_{S R}$ to perform heading changes. While $V_{M C}$ has to be demonstrated with two engines inoperative only at landing where fuel shortage is expected. This is not as constraining as for the take off where a much higher power is needed. A carefully designed architecture would normally avoid the sudden stop of engines located one next to another as in the case of Ampere [21]. Other risks however might damage closely located engines such as bird collision or rupture of a propeller.

For these reasons it has been decided to render at least three engines inoperative, representing one fourth of the total power. Thus the total number of engines for the ATR72 with DEP is fixed to twelve.

A total of four configurations are studied to capture the overall changes that differential thrust and VT reduction bring to the aircraft. These configurations are resumed in table 6 It must be stressed that velocities are calculated from publicly available data and thus are only indicative [37].

The flight conditions represent a rectilinear climb at constant velocity. The same climb angle is imposed for all configuration to allow comparison in an identical flight situation. For each configuration an equilibrium map is built by swapping side slip angle and flight velocity. Along the flight envelop comes the rudder deflection and throttle distribution of a single equilibrium point. Side slip angle and velocity for this point is shown in table 6 
Table 6 Aircraft Configurations

\begin{tabular}{|c|c|c|c|c|}
\hline Configuration & Original & DEP 1 & DEP 2 & DEP 3 \\
\hline Description & Original ATR72 & DEP ATR72 & $\begin{array}{c}\text { DEP ATR72 } \\
\text { Differential Thrust }\end{array}$ & $\begin{array}{c}\text { DEP ATR72 } \\
\text { Differential Thrust } \\
\text { and small VT }\end{array}$ \\
\hline Engines & 2 & 12 & 12 & 12 \\
\hline Inoperative engines & 1 & 3 & 3 & 3 \\
\hline VT area & $S_{v_{0}}$ & $S_{v_{0}}$ & $S_{v_{0}}$ & $0.7 S_{v_{0}}$ \\
\hline Rudder allowed & Yes & Yes & No & No \\
\hline Gradient of climb & $3.0 \%$ & $3.0 \%$ & $3.0 \%$ & $3.0 \%$ \\
\hline Turn rate, $\Omega(\mathrm{rad} / \mathrm{s})$ & 0 & 0 & 0 & 0 \\
\hline \multirow{2}{*}{\multicolumn{5}{|c|}{$\begin{array}{l}\text { Feathered, no drag assumed } \\
\text { Additional parameters }\end{array}$}} \\
\hline & & & & \\
\hline$V_{\text {app }}=1.13 V_{\text {app }}(\mathrm{m} / \mathrm{s})$ & \multicolumn{4}{|c|}{56} \\
\hline$V_{\mathrm{sr}}(\mathrm{m} / \mathrm{s})$ & \multicolumn{4}{|c|}{50.5} \\
\hline $1.3 V_{\mathrm{sr}}(\mathrm{m} / \mathrm{s})$ & \multicolumn{4}{|c|}{65} \\
\hline VT stall limit & \multicolumn{4}{|c|}{$\beta=15^{\circ}$} \\
\hline \multicolumn{5}{|c|}{ Flight parameters for throttle distribution and rudder deflection } \\
\hline$\beta$ & \multicolumn{4}{|c|}{$0^{\circ}$} \\
\hline $\mathrm{V}(\mathrm{m} / \mathrm{s})$ & \multicolumn{4}{|c|}{60} \\
\hline
\end{tabular}




\section{B. Flight envelop map}

\section{Map Interpretation}

The flight envelops along with the accompanying throttle distribution and rudder deflection are shown in Fig 5 to Fig 8 For each equilibrium map, a point indicates an equilibrium. If this equilibrium is on the edge of the stability map a line shows the limiting parameter. It is either the $5^{\circ}$ limitation in roll, stall or rudder saturation. For distributed propulsion, engine saturation is indicated with different markers. A rectangular marker signifies that one engine is saturated, up-triangle two engines, down triangle three engines, left triangle four engines and finally right triangle five engines. Additionally, the complete zone after $\|\beta\| \geq 15^{\circ}$ is faded, signifying that any equilibrium is valid under the condition that the VT did not yet experienced stall.

\section{ATR72 twin engine and Distributed propulsion}

The baseline ATR72 is presented in Fig 5a and the trim inputs in Fig 5b. The climb gradient is slightly unfavorable for this configuration nevertheless, one can observe the compliance with the controlability requirement at low velocity and the $15^{\circ}$ margin at $1.3 V_{S R}$. The limiting parameters for the flight envelop are the roll angle limitation for negative side slip, rudder deflection for positive side slip. The map is not symmetrical as expected for the case of engine failure.

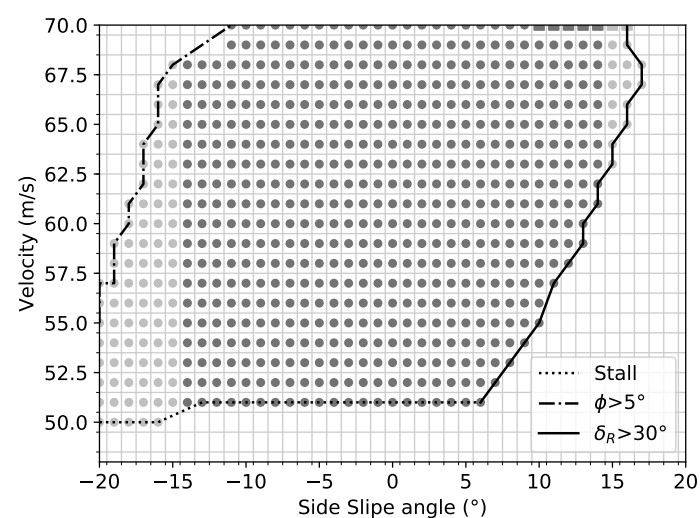

(a) Original ATR72, flight envelop.
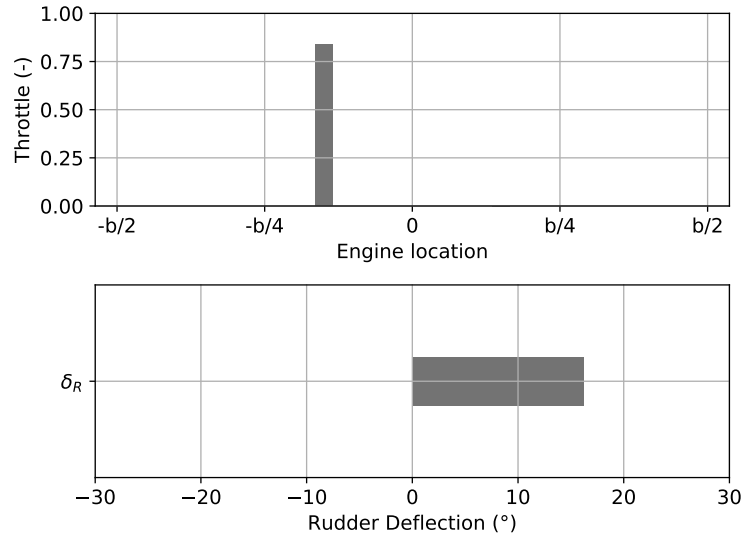

(b) Original ATR72, throttle level and rudder deflection.

Fig. 5 Map of equilibrium points of the Original configuration and throttle level and rudder deflection for trim at $\mathrm{V}=60 \mathrm{~m} / \mathrm{s}, \beta=0$

Fig 6a and Fig 6b show the same airplane equipped with a distributed propulsion however not using differential thrust as shown in Fig 6b. Despite the fact that the power loss is only one fourth of the total power, rudder deflection remains similar to the previous case and the equilibrium map is only increased by a few degrees to the right.

This can be reasonably explained by the fact that the outer most engines on the left wing, despite representing only one fourth of the power, have a high level arm thus creating an important yaw moment. At this stage, designing an aircraft with Distributed Propulsion would not bring substantial reduction on the VT surface area since it remains the primary control effector for yaw. This changes once Differential Propulsion is activated.

\section{ATR72 using differential thrust and small VT}

Fig $7 \mathrm{a}$ and Fig $7 \mathrm{~b}$ respectively show the equilibrium map and the trim inputs for an ATR72 with distributed propulsion and differential thrust activated.

It is important to remember that to segregate the possible increase of control provided by the differential thrust, the rudder isn't activated for the remaining configurations as it is shown in Fig $7 \mathrm{~b}$. Continuing on the same figure, the thrust distribution found by the optimizer is linear except for the saturated engine(s) as it is the case here.

The equilibrium map shows one main characteristic of differential thrust: control power decreases with increasing velocity.

In consequence, since the flight enveloped is increased at low velocity with respect to Fig $5 \mathrm{a}$ and Fig $6 \mathrm{a}$, it can be 


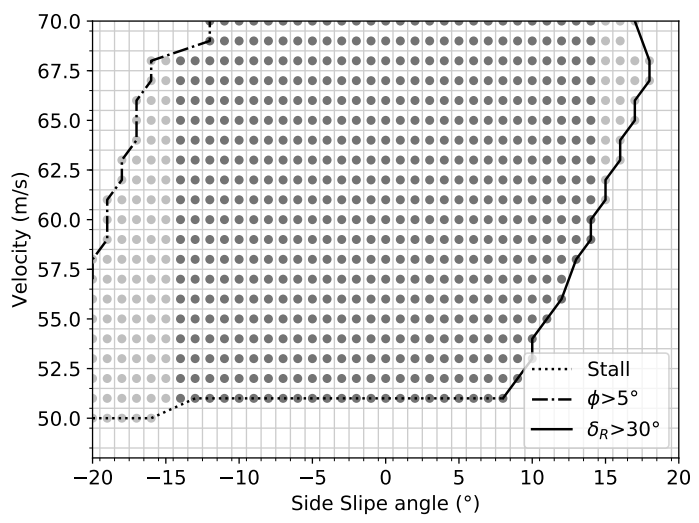

(a) DEP 1 flight envelop.
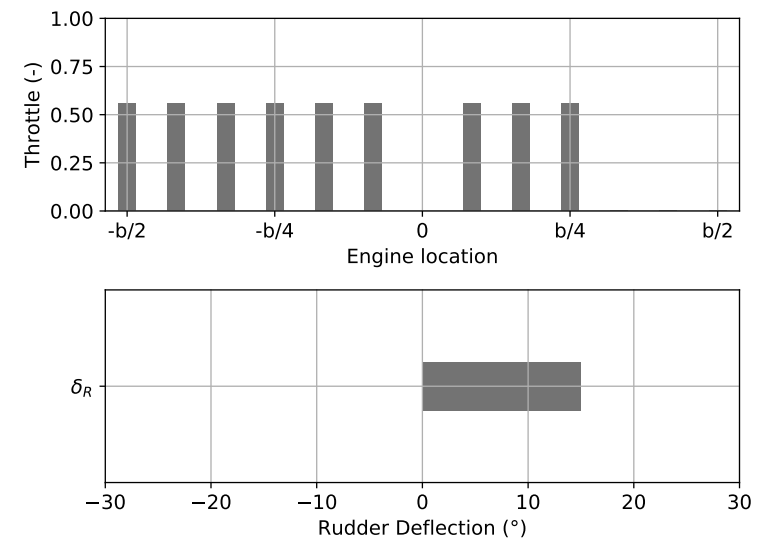

(b) DEP 1, throttle level and rudder deflection.

Fig. 6 Map of equilibrium points of the configuration DEP 1 and throttle level and rudder deflection for trim at $\mathrm{V}=60 \mathrm{~m} / \mathrm{s}, \beta=0$

stated that differential thrust is able to maintain lateral control at low velocity. However at $1.3 V_{S R}$ the control margin is too low.

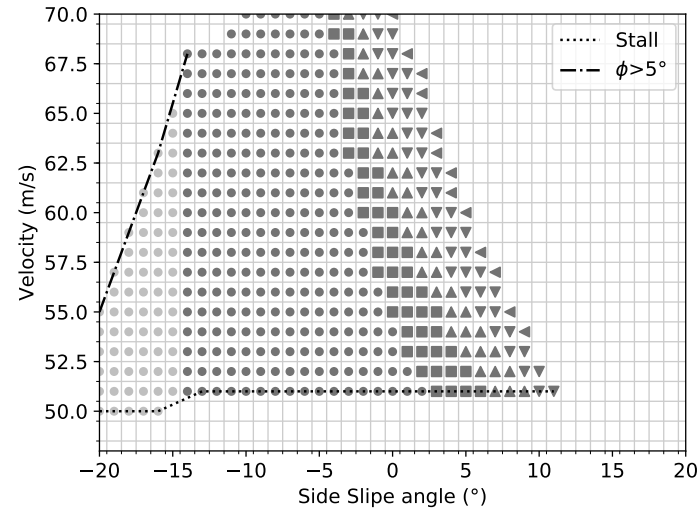

(a) DEP 2, flight envelop.
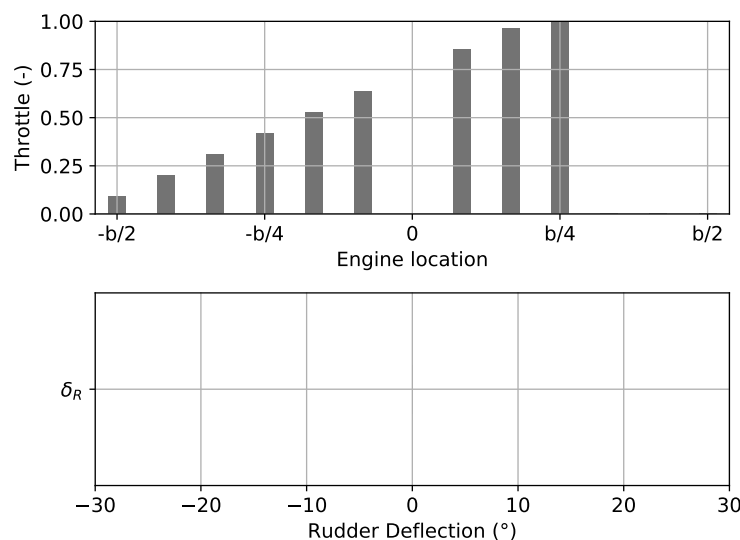

(b) DEP 2, throttle level and Rudder deflection at $60 \mathrm{~m} / \mathrm{s}$ and $\beta=0^{\circ}$.

Fig. 7 Configuration DEP 2 using only Differential Thrust. Rectangles indicate an equilibrium with one engine at saturation (full throttle), for map interpretation see paragraph 1

The main reason why the flight envelop is reduced at high velocity is because the VT is generating a restoring moment as soon as side slip appears. To increase the flight envelop, one simply has to reduced the VT, which is performed in Fig $8 \mathrm{a}$ and Fig $8 \mathrm{~b}$. Here the VT surface area is reduced by $30 \%$, the aircraft remains positively damped, that is $C_{n_{\beta}}>0$, however the equilibrium map improves vastly. The directional control margin at $1.3 V_{S R}$ is now compliant with the certification despite the fact that a higher number of engines are being used at full throttle to maintain equilibrium.

\section{Interpretation and limits of the results}

So far the following observation were made:

- Equipping an ATR72 with distributed propulsion but not differential thrust would not guaranty a reduction of VT surface area due to the important level arm of engines located at the wing tip.

- Differential thrust alone would be sufficient to maintain full control of the aircraft at low velocity due to the high 


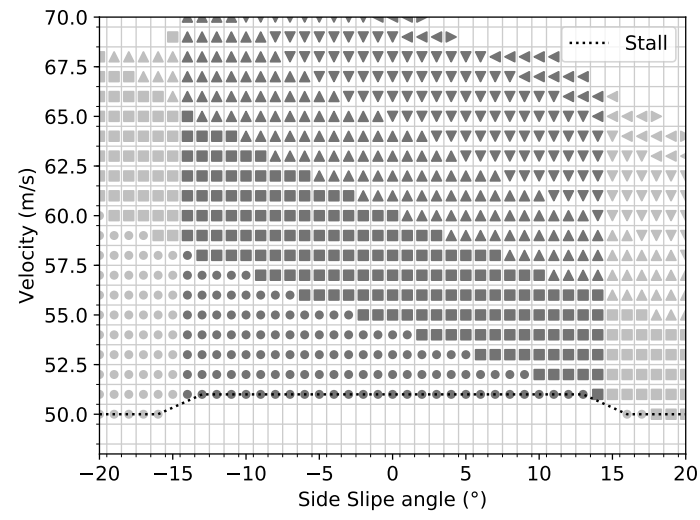

(a) DEP 3, flight envelop
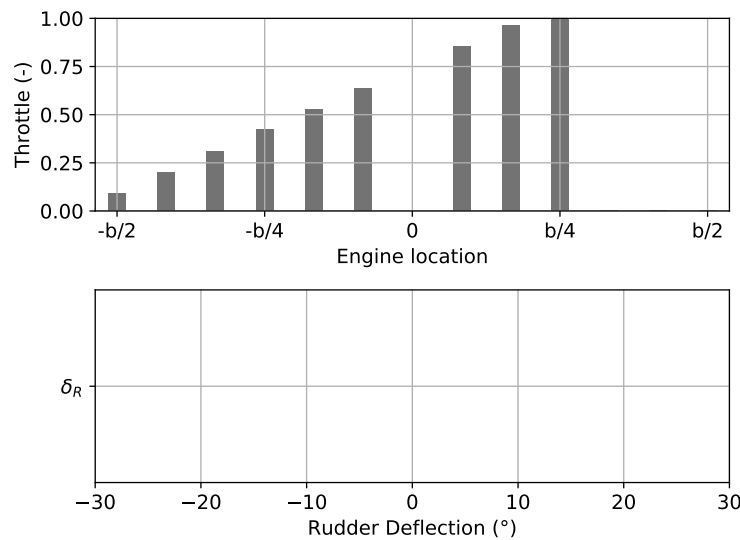

(b) DEP 3, throttle level and rudder deflection at $60 \mathrm{~m} / \mathrm{s}$ and $\beta=0^{\circ}$

Fig. 8 DEP 3, with $S_{v}=0.7 S_{v_{0}}$. Rectangles indicate an equilibrium with one engine at saturation (full throttle), for map interpretation see paragraph 1

control effectiveness.

- The VT actually has to be reduced to be able to exploit the full potential of differential thrust.

Limitations can be anticipated if one extrapolates these results. The reduction of VT surface area could be further increased but then stability at high velocity, when differential thrust loses its control effectiveness would be badly degraded. This is observable as each aircraft employing differential thrust see their equilibrium map reducing with increasing velocity. Nevertheless this shows that employing differential thrust brings a new paradigm for the design of the VT by changing the design case.

The study is limited to the precision of the thrust model employed as well as the important assumption that no interaction takes place between propeller and wing. Models taking these effects into account can now be added to the framework. The framework itself suffers from the under-constrained system of equations mostly when differential thrust and rudder are allowed together. It seems that the objective function doesn't induce enough constraints on the distribution of the yaw moment. The definition of an explicit allocation function could further increase the capability of the framework to predict trim points with differential thrust. Additionally, this study is limited by the aerodynamic models that consider non linear effects only with VT geometry and linear effects otherwise. That is no consideration of stall, efficiency decrease with rudder deflection or masking of VT by the fuselage.

As future work, implementation of interaction models and more precise thrust model has to be performed along with a better formulation for yawing moment distribution. In parallel of such development, the framework allows to study the dynamic behavior of such configurations with or without stability augmentation system. It is also necessary to assess the variation of stability gains observed with the configuration i.d. the total number of engines, the ratio of inoperational engines as well as the total on board power. These will be part of future research in an effort to integrate this work in an multidisciplinary optimization process for preliminary sizing.

\section{Conclusion}

Reduction of Vertical Tail surface area is tackled in this study considering an emerging and game changing technology: Differential Thrust with Distributed Electric Propulsion (DEP). This technology could benefit commuter aircraft as they are envisioned to be the first all electric aircraft for passenger transportation. To assess the flight stability and subsequent handling qualities, a framework relying on a trimming algorithm has been developed allowing to compare a traditional aircraft with one using differential thrust. To take into account the effect of the reduction of Vertical Tail, we could rely on the VeDSC method developed at Naples University. This method increases largely the precision of previous preliminary aerodynamic performance calculation methods such as DATCOM or ESDU by interpolating results of hundreds of high fidelity CFD simulations. Non linear effects parameterized by geometrical parameters are captured and renders our framework extremely versatile to study aircraft of the type of the ATR72. A 
simplified formulation of distributed propulsion, without propeller wing interaction effect is used in order to quickly estimate the potential of differential thrust to control the aircraft.

Results show an important increase of control at low velocity, where propellers are generating the highest thrust while control at high velocity is limited. Interestingly, reducing the Vertical Tail conducts to a dramatic increase of the flight envelop, while relying exclusively on Differential Thrust. Overall, it has been shown that using differential thrust can result in reduction of Vertical Tail as well as a new paradigm for the design of the Vertical Tail. This one would have to be sized for a different flight case most probably at higher velocity, assuring a better efficiency of the Vertical Tail and hence a substantial reduction. Further studies will concern the variation of the performances with the configuration (number of engines, number of inoperational engines etc...) such as to guide the designer who wishes to design an aircraft using Differential Thrust as well as the introduction of a propeller-wing interaction model.

\section{Acknowledgments}

The author would like to thank Mamoun El Oueldrhiri for his support in the exploration of equilibrium with distributed propulsion.

\section{References}

[1] Ciliberti, D., Nicolosi, F., and Vecchia, P. D., “A New Approach in Aircraft Vertical Tailplane Design,” XXII Conference AIDAA, 2013.

[2] Rediess, H. A., "Impact of Advanced Control Concepts on Aircraft Design," $12^{\text {th }}$ ICAS Congress, , No. Paper 80 - $0.4,1980$.

[3] Abzug, M. J., and Larrabee, E. E., Airplane Stability and Control, A History of the Technologies that Made Aviation Possible, 2005.

[4] Anderson, M. R., and Mason, W. H., "An MDO Approach to Control Configured Vehicle Design," AIAA Meeting Papers on Disc, , No. 96-4058, 1996, pp. 734-743.

[5] B. Chudoba, H. S., "A Generic Stability and Control Methodology for Novel Aircraft Conceptual Design,” AIAA Atmospheric Flight Mechanics Conference, , No. 5388, 2003.

[6] Ruben E Perez, H. T. L., and Behdinan, K., "Multidisciplinary Optimization Framework for Control Configuration Integration in Aircraft Conceptual Design,” Journal of Aircraft, Vol. 43, No. 6, 2006.

[7] Jason Welstead, G. L. C. J., "Conceptual Design Optimization of an Augmented Stability Aircraft Incorporating Dynamic Response and Actuator Constraints," 52 ${ }^{\text {nd }}$ AIAA Aerospace Sciences Meeting, , No. AIAA Paper 2014-0187, 2014.

[8] Denieul, Y., "Preliminary Design of Control Surfaces and Laws for Unconventional Aircraft Configurations," Theses, INSTITUT SUPERIEUR DE L'AERONAUTIQUE ET DE L'ESPACE (ISAE), Dec. 2016. URL https://hal . archivesouvertes.fr/tel-01482103

[9] Cozensa, D., and Vos, R., "Handling Qualities Optimization in Aircraft Conceptual Design," $17^{\text {th }}$ AIAA AVIATION Forum, , No. 3763, 2017.

[10] Feuersanger, A. P., “Control of Aircraft with Reduced Stability,” Phd thesis, ISAE-SUPAERO, ONERA, 2008.

[11] Mooney, H. P., Brandt, J. B., Lacy, D. S., and Whalen, E. A., “AFC-Enabled Vertical Tail System Integration Study,” NASA ID $20140003900,2014$.

[12] Nicolosi, F., Vecchia, P. D., and Ciliberti, D., "An investigation on vertical tailplane contribution to aircraft sideforce," Aerospace Science and Technology, Vol. 28, No. 1, 2013, pp. 401 - 416. doi:https://doi.org/10.1016/j.ast.2012.12.006, URL http://www.sciencedirect.com/science/article/pii/S1270963812002052.

[13] “Certification Specifications and Acceptable Means of Compliance for Large Aeroplanes CS-25,", August 2017.

[14] Morris, C. C., "Flight Dynamic Contraints in Conceptual Aircraft Design Analysis and Design Optimization,” Phd thesis, Virginia Polytechnic Institute, 2013.

[15] Della Vecchia, P., "Development of Methodologies for the Aerodynamic Design and Optimization of New Regional Turboprop Aircraft," 2013. 
[16] Lin, J. C., and al, "Innovative Flow Control Concepts for Drag Reduction," 2016 AIAA SciTech Forum and Exposition, , No. NASA ID 20160007668, 2016.

[17] Tucker, T., "Touchdown : the Developement of Propulsion Controlled Aircraft at NASA Dryden,”, 1999.

[18] Moore, M. D., and Fredericks, B., "Misconception of Electric Propulsion Aircraft and their Emergent Aviation Market," 52 $2^{\text {nd }}$ AIAA Aerospace Sciences Meeting, , No. 0535, 2014.

[19] Ko, A., Schetz, J. A., and Mason, W. H., "Assessment of the Potential Advantages of Distributed Propulsion for Aircraft," ISABE-2003-1094, 2003.

[20] Felder, J. L., Kim, H. D., and Brown, G. V., “Turboelectric Distributed Propulsion Engine Cycle Analysis for Hybrid Wing Body Aircraft," $47^{\text {th }}$ AIAA Aerospace Sciences Meeting, , No. 1132, 2009. doi:10.2514/6.2009-1132.

[21] Hermetz, J., Ridel, M., and Döll, C., "Distributed electric propulsion for small business aircraft a concept-plane for key-technologies investigations," CAS 2016, Sep 2016, DAEJEON, South Korea, 2016.

[22] Borer, N. K., Patterson, M. D., Viken, J. K., Moore, M. D., and al, "Design and Performance of the NASA SCEPTOR Distributed Electric Propulsion Flight Demonstrator," $16^{\text {th }}$ AIAA Aviation Technology, Integration, and Operations Conference, , No. 3920, 2016.

[23] Grellet, G., and Clerc, G., Actionneurs électriques, 1997.

[24] Goman, M. G., Khramtsovsky, A. V., and Kolesnikov, E. N., "Evaluation of Aircraft Performance and Maneuvrability by Computation of Attainable Equilibrium Sets," JOURNAL OF GUIDANCE, CONTROL, AND DYNAMICS, Vol. 31, No. 2, 2008.

[25] Boiffier, J. L., The Dynamics of Flight, 1998.

[26] Sachs, G., "Flight Performance Issues of Electric Aircraft," AIAA Atmospheric Flight Mechanics Conference, , No. $4727,2012$.

[27] “Aerospace Standard AS 50881 : Wiring Aerospace Vehicle,”, 2010.

[28] Oppenheimer, M. W., and David B Doman, P., "Control allocation for overactuated systems," 4th Mediterranean Conference on Control Automation proceedings, , No. 321, 2006.

[29] Stückl, S., "Methods for the Design and Evaluation of Future Aircraft Concepts Utilizing Electric Propulsion Systems," Phd thesis, Technische Universität München, 2015.

[30] "Type Certificate data sheet No. A53EU,", 2015.

[31] "Jane's all the World Aircraft,", 2015.

[32] Chudoba, B., "Stability and Control of Conventional and Unconventional Aircraft Configuration," Phd thesis, 2001.

[33] Diederich, F. W., "A planform parameter for correletating certain aerodynamic characteristics of swept wing," , No. NACA-TN$2335,1951$.

[34] Nicolosi, F., Della Vecchia, P., Ciliberti, D., Corcione, S., and Cusati, V., "A Comprehensive Review of Vertical Tail Design," 6th Symposium on Collaboration in Aircraft Design, At Warsaw, 2017.

[35] Ciliberti, D., Vecchia, P. D., Nicolosi, F., and Marco, A. D., "Aircraft directional stability and vertical tail design: A review of semi-empirical methods," Progress in Aerospace Sciences, Vol. 95, 2017, pp. 140 - 172. doi:https://doi.org/10.1016/j.paerosci. 2017.11.001, URL http://wWw.sciencedirect.com/science/article/pii/S0376042117301598

[36] Etkin, B., Dynamics of Atmospheric flight, 1979.

[37] “Independance Virtual Airlines, Speed charts ATR72-500,”, 2006. URL http://www.independance.pl/atr-speeds html 